\title{
Solanum melongena allergy (A comprehensive review)
}

\author{
OVIDIU NICOLAE BERGHI ${ }^{*}$, DANIELA VRINCEANU ${ }^{2 *}$, ROMICA CERGAN $^{3}$, \\ MIHAI DUMITRU $^{3 *}$ and ADRIAN COSTACHE ${ }^{4}$ \\ ${ }^{1}$ Department of Oncologic Dermatology, 'Elias' Emergency University Hospital, 011461 Bucharest; \\ ${ }^{2}$ ENT Department, Bucharest Emergency University Hospital, 010271 Bucharest; ${ }^{3}$ Department of Anatomy, \\ 'Carol Davila' University of Medicine and Pharmacy, 050474 Bucharest; ${ }^{4}$ Pathology Department, \\ 'Carol Davila' University of Medicine and Pharmacy, 020021 Bucharest, Romania
}

Received June 1, 2021; Accepted July 1, 2021

DOI: $10.3892 / \mathrm{etm} .2021 .10495$

\begin{abstract}
The Solanaceae family, including, among other, eggplants, represents the sixth most widely cultivated crops around the globe. We review the current data regarding allergies to Solanum melongena (eggplants), generating symptoms that range from gastrointestinal to respiratory allergic reactions. Currently, there are more than 4 mechanisms and molecules presumably involved in triggering allergic reactions to Solanum melongena: The lipid transfer protein (LTP) pathway, the profilin pathway, polyphenol oxidase (PPO) mechanism and other molecules. Allergies may be triggered both by pollen respiratory reactions and fruit intake. There is also an important cross-reactivity mechanism revealed by recent studies. Our literature review revealed many case series studies, some with in-depth molecular analysis of the triggering mechanism. However, wide population studies are still scarce. Current geographical distribution of the crops and population migrations should enhance the awareness of allergy and immunology specialists, ENT specialists, emergency physicians and pediatricians to the need for proper routine laboratory testing for possible Solanum allergy.
\end{abstract}

\section{Contents}

1. Introduction

2. Lipid transfer protein mechanism

3. Profilin pathway

4. Other mechanisms

5. Importance of polyphenol oxidase

6. Conclusions

Correspondence to: Dr Romica Cergan, Department of Anatomy, 'Carol Davila' University of Medicine and Pharmacy, 8 Eroii Sanitari Boulevard, 050474 Bucharest, Romania

E-mail: r.cergan@gmail.com

*Contributed equally

Key words: Solanum melongena, allergy, lipid transfer protein, profilins, polyphenol oxidase

\section{Introduction}

The Solanaceae family represents a large group of plants with more than 3,000 species. They are adapted to various types of geographical conditions from all continents except for Antarctica. The most important representatives include eggplants (Solanum melongena), tomatoes (S. lycopersicum), potatoes (S. tuberosum), tobacco (Nicotiana tabacum) and petunias (Petunia inflata) (1). Asian eggplants (S.melongena L.) are one of the most important vegetables in the world, ranking at the sixth place of the most cultivated crops around the globe after tomatoes (Solanum lycopersicum L.), watermelons (Citrullus lanatus), onions (Allium cepa L.), cabbage (Brassica oleracea L.) and cucumbers (Cucumis sativus L.) (2). Eggplants are produced mainly in Southeast Asian, African, and Mediterranean areas, especially in China and India (90\% percent of the total production). Indonesia, Turkey, Egypt, the Philippines, and Iran are second-line producers, covering $1 \%$ of the world's total production $(1,3)$. Approximately 54 million tons were produced in 2018 according to FAOSTAT (Food and Agriculture Organization of the United Nations) (3). From a medical point of view, eggplants are considered to present antioxidant capacity, due their high content of phenolics and flavonoids (2).

For this review, PubMed was queried using the key words 'eggplant' and 'allergy' and the search was limited to free full text available articles. All 25 articles identified were included in this review and other specific aspects were clarified using also free full text sources on PubMed or established internet reference websites. Applying other inclusion criteria or diminishing the time frame would have limited the scope of this review. The present review is one of the first attempts to cover all possible immunology mechanism associated with eggplant allergy.

During the last two decades, several studies, including small series of patients and case reports, have presented various aspects concerning the allergy to eggplants. Food allergies were the most frequent reactions to this vegetable, followed by respiratory reactions to pollen.

\section{Lipid transfer protein mechanism}

Lipid transferprotein (LTP) is a well-known stablecross-reacting plant food pan-allergen. LTP syndrome results from a primary 
sensitization to LTPs, inducing frequent systemic reactions and even anaphylaxis (4). LTPs are small and stable proteins, resistant to heat and acid $\mathrm{pH}$, with a rigid tertiary structure formed by four disulphide bridges that carry lipids through the cell walls. These proteins are most frequently present in the surface tissues (peel) of fruits such as apples, peaches, apricots, cherries, plums, pears, raspberries, strawberries, blackberries, and other fruits (5). LTPs have also been described as allergens in the pollens of mugwort (Art v 3), plane tree (Pla a 3), olive (Ole e 7), pellitory (Par j 1-2), cypress and ragweed (Amb a 6) $(4,6)$. Patients with sensitization to non-specific LTPs, mainly due to primary sensitization to peaches (Pru p 3 as the primary sensitizer), can develop cross-sensitization to other fruits and vegetables containing LTPs (5), these findings suggesting that primary sensitization to pollen LTPs may be a cause for plant food LTP allergy by cross-reactivity. The clinical presentation of the LTP syndrome (from local symptoms to anaphylaxis) may be severe in some patients and reactions can be induced by both fresh and processed foods. Peeled fruits are better tolerated, because LTPs accumulate mostly at the surface of the food (4). LTPs have been implicated in some cases of eggplant allergy. Asero et al evaluated 49 patients monosensitized to LTP and found 2 cases (4\%) of allergy to eggplant (7). Ethiopian eggplant (Solanum aethiopicum) represent a fruiting plant of the Solanum genus, mainly found in Asia and Tropical Africa, with cross-reactivity to eggplant. Sixty patients from the Zurich area with pollen allergy (birch, grass and mugwort sensitization) were exposed to three exotic vegetables (water spinach, hyacinth bean, Ethiopian eggplant), unexperienced before, in order to evaluate the presence of proteins related to pan-allergens (Bet $\mathrm{v} 1$ homologous proteins, profilins and LTPs). The binding to LTPs of 8-10 kDa has been observed in the extract of eggplant with anti-Pru p 3 and anti-Cor a 8 . These results were partially confirmed in some patients using skin prick tests and open oral food challenges, with six of them developing an oral allergy syndrome (OAS) under challenge (8). Jain and Salunke isolated, purified, crystallized, and solved the structure of first alike non-specific lipid transfer protein (nsLTP), SM80.2, from eggplant. SM80.2 showed structural similarity in overall structure with known nsLTP structure, but variation in sequence, role and hydrophobic pocket (9). Ukleja-Sokołowska et al described a case of threatening food allergy to eggplant in a male patient known with a history of allergic reactions to salad and chicken with spices. Skin prick tests were positive for eggplant, both cooked and fresh, grass pollen and cat dander. Positive results were obtained in the laboratory for $\mathrm{rPhl} \mathrm{p}$ 1, rCan f 5, Fel d 1 and LTPs-nJug r 3, rPru p 3, rPla a 3 using Immunocap ISAC. Based on the clinical picture, prick tests and laboratory analysis, the authors concluded that the patient suffered from LTP syndrome (10).

\section{Profilin pathway}

Profilins are small ubiquitous proteins and cross-reacting plant food pan-allergens. These allergens have been identified in fruits such as apples, peaches, pears, and strawberries and in vegetables such as celery and carrots (5). Sola $\mathrm{m} 1$ is unique, as it was found to contain a 28 amino acid long stretch (K41-A68). The authors hypothesized that Sola $\mathrm{m} 1$ may be a genuine sensitizer for these patients and not just a cross-reactive protein.
rSola $\mathrm{m} 1$ allergen was localized in the edible seeds of eggplant using in situ immunolocalization studies. Sola $\mathrm{m} 1$ was found to share $>80 \%$ sequence identity with Bet v 2 in IgE-inhibition assay. IgE-cross reactivity was also observed between rSola $\mathrm{m} 1$ and profilins from six other foods (11).

The same team of Jaind and Salunke isolated, purified and crystallized SM80.1 from S. melongena using ammonium sulphate fractionation and size-exclusion chromatography. The protein of size $45 \mathrm{kDa}$ showed homology to $7 \mathrm{~S}$ allergenic vicilins and it was purified from seeds of $S$. melongena. The study identified an important homology of SM80.1 to an allergy-related protein from $S$. lycopersicum, by performing a BLAST search (12).

\section{Other mechanisms}

Three protein bands of relative molecular mass (Mr) of 71, 64, and $60 \mathrm{kDa}$ were detected in the sera of 3 allergic subjects. All three patients presented signs and symptoms of allergy after they ingested baked eggplant. The immunoblot analysis was accustomed to show the presence of these three allergens in eggplant; the 71-kDa allergen looked as if it would be heat stable (13). Two case reports presented allergies to eggplant in patients with latex allergy. Lee et al reported a patient with latex allergy who experienced anaphylaxis after ingesting boiled eggplant. Using SDS-PAGE and immunoblot analysis, three IgE-binding proteins (1 protein with molecular mass between 22 and $36 \mathrm{kDa}, 1$ protein near $36 \mathrm{kDa}$, and 1 protein between 36 and $50 \mathrm{kDa}$ ) were identified in fresh and boiled eggplant extract. One IgE-binding latex protein was detected between 22 and $36 \mathrm{kDa}$ with the identical mass, with the one from the eggplant possibly a candidate allergen to prove cross-reactivity (14). In another case report, Gamboa et al described a patient with facial and hand angioedema, conjunctivitis, generalized pruritus, and glottic difficulty to breath after eating eggplant. The patient presented a history of latex-fruit syndrome after presenting with rhinitis, skin, and pharyngeal pruritus after eating chestnut (first episode) and anaphylaxis after eating banana and subsequently, after minimum environmental exposure to latex, asthma attack, rhinitis, conjunctivitis, and urticaria (second episode). Fruit class I chitinases with an $\mathrm{N}$-terminal heveinlike domain and latex hevein (Hev b 6.02) are identified in the literature as the main allergens liable for cross-reactions. Hev b 6.02 (latex hevein) and Prs a 1 (avocado class I chitinase) are utilized in in vitro and in vivo assays as controls. Hev b 6.02 was found to produce positive skin responses and showed high biological activity in histamine release (HRT) and basophil activation test (BAT). Prs a 1 reached high values of basophil activation and over $10 \%$ of histamine release (15). Pramod and Venkatesh described a case of allergy caused by ingestion of eggplant in an atopic subject, due to a low molecular weight non-protein secondary metabolite of less than $1 \mathrm{kDa}$ using size-exclusion chromatography of the filtrate of eggplant extract on Sephadex G-25 followed by skin prick test (SPT) of the fractions (16).

\section{Importance of polyphenol oxidase}

Polyphenol oxidase (PPO) is an extremely important eggplant enzyme. PPOs are involved in plant defense almost like 
allergenic proteins of the PR family (chitinases, glucanases and peroxidases). The analysis of tryptic peptides of eggplant $64 \mathrm{kDa}$ protein bands showed significant similarity in peptide matches to the eggplant PPO sequences available in the database. Six cases of eggplant allergy were confirmed by positive SPT and the $64 \mathrm{kDa}$ protein was confirmed as an allergen (Sola m PPO4), based on the presence of specific bound copper, detection by polyclonal sweet potato PPO antiserum and bioinformatic analysis including prediction of the $\operatorname{IgE}$ epitope (17). Six eggplant-allergic subjects were assessed for sensitization to eggplant (peel/pulp and raw/cooked) by SPT, allergen-specific IgE, and immunoblots by Babu and Venkatesh in 2009. SPTs with peel, pulp, raw and cooked eggplant extracts were positive for all eggplant-sensitized subjects; allergen-specific IgE was also positive to all components tested. Raw eggplant contains 5 allergens in the $36-71 \mathrm{kDa}$ range, most allergens being localized in the eggplant peel than the pulp; some seem to be heat stable. Four of them were detected as glycoproteins and three displayed retention of IgE-binding ability in simulated gastric fluid digestion (18). The prevalence of IgE-mediated eggplant allergy was estimated at $0.8 \%$, with higher rates of sensitization in females (19).

Apart from the cases of food allergy to eggplant, a case report from 2002 described a 43-year-old male smoker from Portugal with recent onset of rhinoconjunctivitis and a dry cough when working in a greenhouse where eggplants were cultivated. SPT and a conjunctival challenge test with a saline extract from Solanum melongena flower petals and pollen were positive. SPT with eggplant fruit was negative (20).

Given these complex molecular interactions, the clinical presentation ranges from skin reactions to possible fatal respiratory distress requiring admittance into the ENT department or in severe cases, transfer to the intensive care unit $(21,22)$.

Although the first suspicion of a possible allergic interaction between eggplant and tobacco was mentioned almost 40 years ago, this domain of activity needs much more effort from the scientific community (23).

Moreover, one of the first attempts to desensitize patients allergic to eggplants dates back to 2001, through the use of a water extract of Solanum melongena (SMWE) (24).

Dermatology is another specialty encountering severe allergic response due to contact dermatitis to eggplant $(25,26)$.

There are still debates regarding the exposure of the pediatric population to eggplant; progressive exposure during childhood seems to prevent extreme allergic response in adolescence, which could lead to tracheostomy and other complex procedures for securing the airways $(27,28)$.

Clinical research is hindered by the inconsistency of the prick tests for eggplant due to the amount of histamine present in eggplant that does not produce a positive SPT response in a majority of non-atopic subjects, and that a positive response in a few subjects are likely to be misdiagnosed as eggplant allergy (29). This research was conducted in India and supposed populations with a genetic background from the Indian peninsula, such as Roma communities, could exhibit the same reaction (30).

Another possible aspect to be taken into consideration is the bacterial wilt of Solanaceae caused by Ralstonia solanacearum Race 1 Biovar 3, and the coexistence of this pathogen in the soil could be the actual immunological trigger in workers harvesting eggplants (31).

At the forefront of the research in this type of allergy, there is the transcriptome analysis of eggplant fruit performed to map epitopes of monoclonal antibodies and polyclonal sera from patients (32).

Previous research proposed the hypothesis that eggplant allergens have a heat-stable nature, as $\operatorname{IgE}$ was reactive to both raw and cooked eggplant extracts (33).

The number of cases with this type of allergic reaction could possibly grow, as beer manufacturers increase the biological value of the beverage by adding antioxidant-rich eggplant (Solanum melongena L.) peel extract (34).

Unfortunately, recent cases present multiple complex allergies and require sublingual immunotherapy as the last line of treatment (35).

\section{Conclusions}

There are eight foods (cow's milk, eggs, tree nuts, peanuts, shellfish, wheat, soy, and fish) that account for $90 \%$ of all food allergies. Particular foods may be consumed preferentially in some parts of the globe and, consequently, they may represent an important cause of food allergy in these populations. Eggplant is an example. A physician, especially an allergist, must pay attention to all details when investigating a case of possible food allergy in a patient when the classic allergens are ruled out. This review underlines that there are at least four pathways and molecules involved in producing Solanum melongena allergy that require further investigations. This type of allergy should draw the attention of medical practitioners, ranging from immunologists to emergency specialists, ENT surgeons or pediatricians.

\section{Acknowledgements}

Professional editing, linguistic and technical assistance performed by Irina Radu, Individual Service Provider.

\section{Funding}

No funding was received.

\section{Availability of data and materials}

All information presented in this review is documented by relevant references.

\section{Authors' contributions}

ONB, DV and MD contributed substantially to the conception and design of the review, the acquisition, analysis, and interpretation of the literature data, and were involved in the drafting of the manuscript. RC contributed substantially to the acquisition, analysis and interpretation of the literature data and were involved in the drafting of the manuscript. AC contributed substantially to the acquisition of the literature data and was involved in the critical revisions of the manuscript for important intellectual content. All authors agreed to be accountable for all aspects of the work in ensuring that 
questions related to the accuracy or integrity of any part of the work are appropriately investigated and resolved. All the authors read and approved the final version of the manuscript for publication.

\section{Ethics approval and consent to participate}

Not applicable.

\section{Patient consent for publication}

Not applicable.

\section{Competing interests}

The authors declare that they have no competing interests.

\section{References}

1. Wei Q, Wang J, Wang W, Hu T, Hu H and Bao C: A high-quality chromosome-level genome assembly reveals genetics for important traits in eggplant. Hortic Res 7: 153, 2020.

2. Rakha M, Namisy A, Chen JR, El-Mahrouk ME, Metwally E, Taha N, Prohens J, Plazas M and Taher D: Development of interspecific hybrids between a cultivated eggplant resistant to bacterial wilt (Ralstonia solanacearum) and eggplant wild relatives for the development of rootstocks. Plants (Basel) 9: 1405, 2020.

3. FAO: Food and Agriculture Organization of the United Nations. FAO-STAT Production Databases. http://www.faostat3.fao.org Accessed August 3, 2020.

4. Fernández-Rivas M: Fruit and vegetable allergy. Chem Immunol Allergy 101: 162-170, 2015.

5. Calamelli E, Liotti L, Beghetti I, Piccinno V, Serra L and Bottau P: Component-resolved diagnosis in food allergies. Medicina (Kaunas) 55: 498, 2019.

6. Palacín A, Gómez-Casado C, Rivas LA, Aguirre J, Tordesillas L, Bartra J, Blanco C, Carrillo T, Cuesta-Herranz J, de Frutos C, et al: Graph based study of allergen cross-reactivity of plant lipid transfer proteins (LTPs) using microarray in a multicenter study PLoS One 7: e50799, 2012.

7. Asero R, Mistrello G, Roncarolo D and Amato S: Detection of some safe plant-derived foods for LTP-allergic patients. Int Arch Allergy Immunol 144: 57-63, 2007.

8. Gubesch M, Theler B,Dutta M,Baumer B, Mathis A,Holzhauser T, Vieths S and Ballmer-Weber BK: Strategy for allergenicity assessment of 'natural novel foods': Clinical and molecular investigation of exotic vegetables (water spinach, hyacinth bean and Ethiopian eggplant). Allergy 62: 1243-1250, 2007.

9. Jain A and Salunke DM: Crystal structure of nonspecific lipid transfer protein from Solanum melongena. Proteins 85: $1820-1830,2017$

10. Ukleja-Sokołowska N, Gawrońska-Ukleja E,Żbikowska-Gotz M, Sokołowski $Ł$ and Bartuzi Z: Recurrent anaphylaxis in patient allergic to eggplant-a lipid transfer protein (LTP) syndrome. Asian Pac J Allergy Immunol 36: 109-112, 2018.

11. Maity S, Bhakta S, Bhowmik M, Sircar G and Bhattacharya SG: Identification, cloning, and immunological studies on a major eggplant (Solanum melongena L.) allergen Sola m 1: A new member of profilin allergen family. Mol Immunol 118: 210-221, 2020.

12. Jain A and Salunke DM: Purification, identification and preliminary crystallographic studies of an allergenic protein from Solanum melongena. Acta Crystallogr F Struct Biol Commun 71: 221-225, 2015.

13. Pramod SN and Venkatesh YP: Allergy to eggplant (Solanum melongena). J Allergy Clin Immunol 113: 171-173, 2004.

14. Lee J, Cho YS, Park SY, Lee CK, Yoo B, Moon HB and Park HS Eggplant anaphylaxis in a patient with latex allergy. J Allergy Clin Immunol 113: 995-996, 2004.
15. Gamboa PM, Sánchez-Monge R, Díaz-Perales A, Salcedo G, Ansótegui J and Sanz ML: Latex-vegetable syndrome due to custard apple and aubergine: New variations of the hevein symphony. J Investig Allergol Clin Immunol 15: 308-311, 2005.

16. Pramod SN and Venkatesh YP: Allergy to eggplant (Solanum melongena) caused by a putative secondary metabolite. J Investig Allergol Clin Immunol 18: 59-62, 2008.

17. Harish Babu BN, Wilfred A and Venkatesh YP: Emerging food allergens: Identification of polyphenol oxidase as an important allergen in eggplant (Solanum melongena L.). Immunobiology 222: 155-163, 2017.

18. Babu BN and Venkatesh YP: Clinico-immunological analysis of eggplant (Solanum melongena) allergy indicates preponderance of allergens in the peel. World Allergy Organ J 2: 192-200, 2009.

19. Harish Babu BN, Mahesh PA and Venkatesh YP: A cross-sectional study on the prevalence of food allergy to eggplant (Solanum melongena L.) reveals female predominance. Clin Exp Allergy 38: 1795-1802, 2008.

20. Gil M, Hogendijk S and Hauser C: Allergy to eggplant flower pollen. Allergy 57: 652, 2002.

21. Rotaru M, Iancu GM, Gheucă Solovăstru L, Glaja RF, Grosu F, Bold A and Costache A: A rare case of multiple clear cell acanthoma with a relatively rapid development of the lower legs. Rom J Morphol Embryol 55 (Suppl 3): S1171-S1179, 2014.

22. Vrinceanu D, Dumitru M, Stefan A, Neagos A, Musat G and Nica EA: Severe DRESS syndrome after carbamazepine intake in a case with multiple addictions: A case report. Exp Ther Med 20: 2377-2380, 2020.

23. Becker CG, Dubin T and Wiedemann HP: Hypersensitivity to tobacco antigen. Proc Natl Acad Sci USA 73: 1712-1716, 1976.

24. Lee YM, Jeong HJ, Na HJ, Ku JY, Kim DK, Moon G, Chae HJ, Kim HR and Kim HM: Inhibition of immunologic and nonimmunologic stimulation-mediated anaphylactic reactions by water extract of white eggplant (Solanum melongena). Pharmacol Res 43: 405-409, 2001.

25. Kabashima K and Miyachi Y: Contact dermatitis due to eggplant. Contact Dermatitis 50: 101-102, 2004.

26. Palla S, Srinivasan AR, Subramanian K and Shekar V: Oral allergy syndrome associated with eggplant particles manifesting as lichenoid reaction-a sequential diagnostic workout and long-term follow up. Indian J Dermatol 65: 550-552, 2020.

27. Chatzi L, Torrent M, Romieu I, Garcia-Esteban R, Ferrer C, Vioque J, Kogevinas M and Sunyer J: Diet, wheeze, and atopy in school children in Menorca, Spain. Pediatr Allergy Immunol 18: 480-485, 2007.

28. Rozsasi A, Neagos A, Nolte F, Riechelmann H, Rettinger G and Keck T: Critical analysis of complications and disorders in wound healing after tracheostomy in children. Laryngorhinootologie 82: 826-832, 2003 (In German).

29. Kumar MN, Babu BN and Venkatesh YP: Higher histamine sensitivity in non-atopic subjects by skin prick test may result in misdiagnosis of eggplant allergy. Immunol Invest 38: 93-103, 2009.

30. Enache G, Rusu E, Ilinca A, Rusu F, Costache A, Jinga M, Pănuş C and Radulian G: Prevalence of overweight and obesity in a Roma population from southern Romania-Calarasi county. Acta Endocrinol (Buchar) 14: 122-130, 2018.

31. Thera AT, Jacobsen BJ and Neher OT: Bacterial wilt of solanaceae caused by Ralstonia solanacearum Race 1 biovar 3 in mali. Plant Dis 94: 372, 2010.

32. Ramesh KR, Hemalatha R, Vijayendra CA, Arshi UZ, Dushyant SB and Dinesh KB: Transcriptome analysis of Solanum melongena L. (eggplant) fruit to identify putative allergens and their epitopes. Gene 576: 64-71, 2016.

33. Hoseini-Alfatemi SM, Bayry J and Sharifi-Rad J: IgE response to two new allergen proteins of Solanum melongena L. (eggplant). Immunol Lett 168: 268-270, 2015.

34. HorincarG,EnachiE,BoleaC,Râpeanu GandAprodu I: Value-added lager beer enriched with eggplant (Solanum melongena L.) peel extract. Molecules 25: 731, 2020.

35. García-Gutiérrez I, Medellín DR, Noguerado-Mellado B, Lillo Ordoñez MC, Abreu MG, Nogales LJ and Rojas-Pérez-Ezquerra P: Treatment with lipid transfer protein sublingual immunotherapy: Slowing down new sensitizations. Asia Pac Allergy 11: e6, 2021. 